\title{
Epidemiological Characteristics of Rodents and Chiggers with Orientia Tsutsugamushi in the Republic of Korea
}

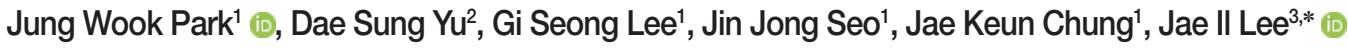 \\ ${ }^{1}$ Division of Infectious Disease Investigation, Health and Environment Research Institute of Gwangju, Gwangju 61986, Korea; ${ }^{2}$ Division of Animal \\ Disease Control, Animal and Plant Quarantine Agency, Gyeongsangbuk-do 39660, Korea; ${ }^{3}$ Department of Veterinary Public Health, College of \\ Veterinary Medicine, Chonnam National University, Gwangju 62287, Korea
}

\begin{abstract}
A survey of rodents and chiggers associated with Orientia tsutsugamushi was conducted in a rural region of the Republic of Korea (Korea) between 2014 and 2018. Overall Apodemus agrarius 15.2\% had the highest seropisitive for O. tsutsugamushi, followed by Myodes regulus 11.4\%. Monthly risk factors using logistic regression analysis were not associated with 0 . tsutsugamushi infections in rodents. The overall prevalence rate of $O$. tsutsugamushi among chiggers was $0.3 \%$. The chigger (Leptotrombidium scutellare) and monthly (October) risk factors were associated with $O$. tsutsugamushi human infections $(P<0.05)$. Orientia tsutsugamushi infections are endemic in rodents in Korea and people, for example, soldiers who are active outdoors, must employ preventive measures, especially during October $(P<0.05)$. When there are many reports of $O$. tsutsugamushi infections in Korea. The Boryong strain $85.7 \%(2 / 14)$ was the most common strain detected in chiggers, followed by the Shimokoshi 7.1\% (1/14) and Karp 7.1\% strains.
\end{abstract}

Key words: Orientia tsutsugamushi, wild rodent, chigger, phylogenetic analysis

\section{INTRODUCTION}

Orientia tsutsugamushi, the causative agent of scrub typhus, is transmitted by chiggers (larval trombiculid mites), and can result in death if untreated. Additionally, chigger bites cancause severe itching in people spending time outdoors, especially during fall [1]. Scrub typhus is distributed throughout much of East and Western Central Asia to northern Australia; cases have also been reported in Africa and Europe [2]. Seasonal outbreaks of scrub typhus in Asia are more frequently reported in the fall and early winter in Korea [3]. Studies on scrub typhus prevalence during fall, the primary period of transmission, have primarily focused on chiggers as vectors of $O$. tsutsugamushi and their distribution [4-7].

The geographic distribution of scrub typhus depends on the population density and vector competence of local chigger vectors [6]. Statistical studies aimed at predicting and preventing the occurrence of scrub typhus infections have been con-

\footnotetext{
- Received 16 February 2020, revised 9 September 2020, accepted 26 September 2020. *Corresponding author (jaeil@chonnam.ac.kr)

ducted both inside and outside Korea with respect to human outdoor activities and to the time spent on activities such as preventive measures as well as the duration of exposure in chigger habitats such as grasses [8,9]. A statistical model was recently presented for scrub typhus occurrence and included climatic factors such as precipitation and humidity, and the occurrence of scrub typhus cases [9]. It is thus necessary to understand the epidemiological parameters for the seasonal prevalence of $O$. tsutsugamushi infections, based on vector distributions and population densities to predict the occurrence of exposure-associated infections [17]; however, there have been few such studies in Korea.

Therefore, this study examined the seasonal trends of scrub typhus and established the appropriate preventive measures. It also includes a cross-sectional study to determine the risk factors associated with the relationship between the occurrence of $O$. tsutsugamushi infections in rodents and the associated chigger vectors from 2014 to 2018 using a statistical program (SPSS 20, IBM, Armonk, New York, USA).

\section{MATERIALS AND METHODS}

\author{
Ethics statement \\ All procedures were conducted under an animal use proto-
}


col approved by the Chosun University Animal Ethics Committee (CIACUC 2016-S0003).

\section{Rodent and chigger collections}

Rodents were collected monthly from a suburban area $\left(35^{\circ} 13^{\prime} \mathrm{N} 126^{\circ} 54^{\prime} \mathrm{E}\right)$ with uncut grasses using Sherman live traps $(3 \times 3.5 \times 9$ inches) (BioQuip, Rancho Dominguez, California, USA). The study area was located in a densely populated area in the southwestern part of Korea, where scrub typhus patients are reported frequently. The information for the human infection rate was obtained from Korea Centers for Disease Control and Prevention(K-CDC) Disease Web Statistics System [3]. In total, 100 traps baited using crackers covered with peanut butter were set monthly from 10:00 to 14:00 hr the following day. Traps positive for rodents were numbered sequentially, placed in a secure container, and then transported to a central laboratory [4]. The rodents were euthanized using chloroform (Merck, New Jersey, USA) soaked in cotton $(1 \times 1 \mathrm{~cm})$, and blood was collected by cardiac puncture using a 10-cc syringe. The collected blood was centrifuged at 3,000 rpm for $20 \mathrm{~min}$, serum was separated, and then stored at $4^{\circ} \mathrm{C}$. An indirect immunofluorescence assay was performed for antibody detection at $24 \mathrm{hr}$ after the rodents were euthanized. After autopsy, the rodents were hung upside down over a dish containing distilled water for $24 \mathrm{hr}$. Chiggers were then collected from the water surface using a fine brush.

\section{Indirect immunofluorescence assay for Orientia tsutsugamushi in wild rodents}

In total, $10 \mu \mathrm{l}$ of serum from each rodent was used. Antigen spot slides for $O$. tsutsugamushi were provided by the Korea Centers for Disease Control and Prevention (KCDC), and indirect immunofluorescence assays were conducted as described previously [14].

Nested polymerase chain reaction(nPCR) for amplifying the 56-kDa TSA gene of $O$. tsutsugamushi in chiggers

Nested polymerase chain reaction (nPCR) was performed as described previously for $O$. tsutsugamushi detection in chigger mites [11]. A total of 30 chiggers/rodent were examined and placed individually on glass slides with $20 \mu \mathrm{l}$ of PBS. The abdomen of each chigger was gently pressed using 2 fine needles until the internal contents were expelled and then observed after suspension in $30 \mu \mathrm{l}$ of PBS under a stereomicroscope (Carl Zeiss, Oberkochen, Germany). The chiggers were mount- ed with polyvinyl alcohol medium (BioQuip) and identified using a morphological key [12]. DNA was extracted from 20 $\mu \mathrm{l}$ of chigger material using a G-spin Total DNA Extraction Kit (iNtRON Biotechnology, Daejeon, Korea). and then analyzed by PCR for the O. tsutsugamushi 56-kDa TSA gene as described previously $[13,14]$.

\section{Risk factors for $O$. tsutsugamushiin rodent hosts}

This study was conducted to identify the relationship between the risk factors (month, species) and prevalence of $O$. tsutsugamushi infection in rodents and chiggers. The month associated with the mean distribution of disease was evaluated as a risk factor in rodents by logistic regression analysis using SPSS version 20 software (IBM).The 95\% confidence interval (CI), and the chi-square analysis was performed for the rodent species and proportion of infection by month as the risk factors in chiggers $(P<0.05)$.

\section{Sequence and phylogenetic analysis}

Amplified PCR products were submitted to Cosmogenetech (Daejeon, Korea) for sequencing using an ABI 3730XL DNA analyzer (Applied Biosystems, Foster City, California, USA). For phylogenetic tree construction, the twelve sequences (KX363949-KX363951, KX363952-KX363953, KY266824KY266830) examined by Park in previous studies $[13,14]$ and 2 sequences (MK451703 and MK451704) that were newly identified in 2017 were compared with O. Tsutsugamushi sequences downloaded from the National Center for Biotechnology Information database, and phylogenetic analyses were conducted using MEGA X. A neighbor-joining tree with 1,000 bootstrap replicates was constructed using the Kimura 2-parameter model.

\section{RESULTS}

In total, 99/652 (15.2\%) serum samples of Apodemus agrarius were seropositive for $O$. tsutsugamushi, and s were positive for O. tsutsugamushi antibodies throughout the year, with the highest seropositive rate observed during October (Table 1). The summer months of July (odds ratio, 1.156; $95 \% \mathrm{CI}, 0.379$ 3.528) $(P=0.800)$ and August (odds ratio, 2.022; 95\% CI, 0.472 $8.657)$ were not associated with seropositivity.

In total, 4,646/10,732 (42.3\%) of the chiggers collected from the 723 rodents collected were individually tested for $O$. tsutsugamushi; of these,14/4,646 (0.3\%) chiggers were positive 
for O. tsutsugamushi (Table 2). In total, 4,170/9,597 (43.4\%) of the chiggers collected from 652 Apodemus agrarius, of which 13 were positive for $O$. tsutsugamushi; only 1 (Leptotrombidium orientale) of 476/1,135 (41.9\%) of the chiggers collected from 35 Myodes regulus was positive for $O$. tsutsugamushi. No chiggers were collected from38 Crocidura lasiura. The 56-kDa TSA gene of O. tsutsugamushi was detected in 12 Leptotrombidium scutellare individuals and one individual each of $L$. orientale and $L$. palpale. The mean number of chiggers collected monthly (except July) from Apodemus agrarius increased significantly beginning in September, and sharply declined in December (Fig. 1).

The results of a chi-squared analysis, by month and species

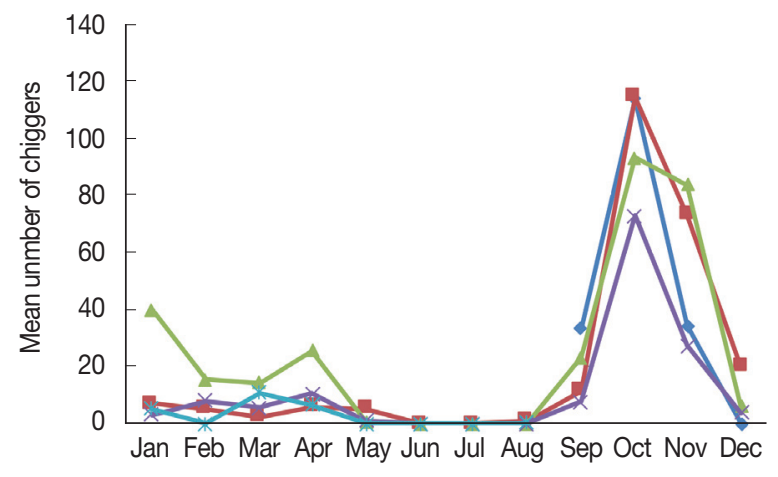

Fig. 1. Monthly mean number of chiggers collected from Apodemus agrarius in Korea from September 2014 to August 2018.

Table 1. The number and percents of Apodemus agrarius, by month from September 2014 to August 2018 that were seropositive for Orientia tsutsugamushi)

\begin{tabular}{lccccc}
\hline Month & No. of rodent & Percent & P-value (0.05) & Odds ratio & 95\% confidence interval \\
\hline January & 37 & 40.0 & 0.032 & 0.308 & $0.105-0.901$ \\
February & 40 & 15.0 & 0.489 & 0.667 & $0.212-2.098$ \\
March & 55 & 5.5 & 0.555 & 0.767 & $0.238-2.159$ \\
April & 42 & 2.4 & 0.404 & 0.615 & $0.197-1.924$ \\
May & 90 & 11.1 & 0.333 & 0.619 & $0.235-1.634$ \\
June & 86 & 15.1 & 0.444 & 0.676 & $0.248-1.842$ \\
July & 72 & 9.7 & 0.800 & 1.156 & $0.379-3.528$ \\
August & 42 & 7.1 & 0.343 & 2.022 & $0.472-8.657$ \\
September & 39 & 15.4 & - & - \\
October & 34 & 47.0 & 0.017 & 0.283 & $0.101-0.796$ \\
November & 53 & 15.1 & 0.160 & 0.490 & $0.181-1.325$ \\
December & 62 & 20.1 & 0.057 & 0.393 & $0.151-1.027$ \\
Total & 652 & 15.2 & &
\end{tabular}

${ }^{*}$ Reference category.

Table 2. The total number and percent of chiggers, by species, positive (\%) for Orientia tsutsugamushi in the Republic of Korea from September 2014 to August 2018

\begin{tabular}{|c|c|c|c|c|c|c|c|c|c|c|}
\hline \multirow{2}{*}{ Total } & & \multicolumn{9}{|c|}{ Species of chigger ${ }^{*}$} \\
\hline & & L. scu & L. ori & L.pal & L. palp & L. zet & N. gar & N. jap & E. kor & W. fra \\
\hline No.chiggers & 4,646 & 2,065 & 1,053 & 734 & 301 & 71 & 51 & 9 & 290 & 72 \\
\hline No. positive & {$[14]$} & [12] & [1] & {$[0]$} & [1] & [0] & {$[0]$} & {$[0]$} & {$[0]$} & {$[0]$} \\
\hline Percent & 0.3 & 0.58 & 0.09 & 0 & 0.3 & 0 & 0 & 0 & 0 & 0 \\
\hline A. agrarius (652) & 4,170 & 1,974 & 782 & 732 & 270 & 67 & 51 & 9 & 285 & 0 \\
\hline No. positive & [13] & [12] & {$[0]$} & {$[0]$} & [1] & [0] & [0] & {$[0]$} & {$[0]$} & [0] \\
\hline Percent & 0.31 & 0.61 & 0 & 0 & 0.4 & 0 & 0 & 0 & 0 & 0 \\
\hline M. regulus (35) & 476 & 91 & 271 & 2 & 31 & 4 & 0 & 0 & 5 & 72 \\
\hline No. positive & [1] & {$[0]$} & [1] & {$[0]$} & [0] & [0] & {$[0]$} & {$[0]$} & {$[0]$} & [0] \\
\hline Percent & 0.21 & 0 & 0.37 & 0 & 0 & 0 & 0 & 0 & 0 & 0 \\
\hline C. lasiura (38) & 0 & 0 & 0 & 0 & 0 & 0 & 0 & 0 & 0 & 0 \\
\hline No. positive & [0] & [0] & [0] & [0] & [0] & [0] & {$[0]$} & [0] & {$[0]$} & [0] \\
\hline Percent & 0 & 0 & 0 & 0 & 0 & 0 & 0 & 0 & 0 & 0 \\
\hline
\end{tabular}

*L. scu: Leptotrombidium scutellare, L. ori: L. orientale, L. pal: L. palpale, L. zet: L. zetum, N.gar: Neotrombicular gardellai, N. jap: N. japonica, E. kor: Euchoengastia koreaensis, W. fra: Walchia fragilis. 
Table 3. The number and percents of rodents and soricomorph that were antibody positive for Orientia tsutsugamushi and relative host factors from September 2014 to August 2018 in the Republic of Korea

\begin{tabular}{lccccc}
\hline Host & $\begin{array}{r}\text { No. of } \\
\text { Tested }\end{array}$ & $\begin{array}{c}\text { No. of } \\
\text { Positive } \\
(\%)\end{array}$ & $\begin{array}{c}P \text {-value } \\
(0.05)\end{array}$ & $\begin{array}{c}\text { Relative } \\
\text { risk }\end{array}$ & $\begin{array}{c}95 \% \\
\text { confidence } \\
\text { interval }\end{array}$ \\
\hline $\begin{array}{c}\text { Apodemus } \\
\text { agrarius }\end{array}$ & 652 & $98(15.1)$ & 0.204 & 1.826 & $\begin{array}{c}0.711- \\
4.689\end{array}$ \\
$\begin{array}{c}\text { Myodes } \\
\text { regulus }\end{array}$ & 35 & $4(11.4)$ & - & - & - \\
$\begin{array}{c}\text { Crocidura } \\
\text { lasiura }\end{array}$ & 21 & $1(4.8)$ & - & - & - \\
\begin{tabular}{c} 
Total \\
\hline
\end{tabular} & 708 & - & & & \\
\hline
\end{tabular}

Table 4. The number and percents of chiggers, by species, colleceted from $A$. agrarius and $M$. regulus in the Republic of Korea from September 2014 to August 2018, which were positive for Orientia tsutsugamushi

\begin{tabular}{lrcccc}
\hline Speices & $\begin{array}{r}\text { No. of } \\
\text { tested }\end{array}$ & $\begin{array}{c}\text { No. of } \\
\text { Positive } \\
(\%)\end{array}$ & $\begin{array}{c}\text { P-value } \\
(0.05)\end{array}$ & $\begin{array}{c}\text { Relative } \\
\text { risk }\end{array}$ & $\begin{array}{c}95 \% \\
\text { confidence } \\
\text { interval }\end{array}$ \\
\hline L.scutellare & 2,065 & $12(0.58)$ & 0.002 & 7.537 & $\begin{array}{c}1.685- \\
33.715\end{array}$ \\
L. orientale & 1,053 & $1(0.09)$ & - & 1 & - \\
L.palpale & 301 & $1(0.88)$ & - & 1 & - \\
L. pallidum & 734 & 0 & - & 1 & - \\
L. zetum & 71 & 0 & - & 1 & - \\
N. gardellai & 51 & 0 & - & 1 & - \\
N. japonica & 9 & 0 & - & 1 & - \\
E. koreaensis & 290 & 0 & - & 1 & - \\
W. fragilis & 72 & 0 & - & 1 & - \\
Total & 4,646 & - & - & - & - \\
\hline
\end{tabular}

as risk factors for O. tsutsugamushi infection are shown in Table 3. Apodemus agrarius was the predominant rodent collected and was not associated (relative risk, 1.826; 95\% CI, 0.7114.689) $(P=0.204)$ with $O$. tsutsugamushi infections. The predominant chigger species, $L$. scutellare, was associated with scrub typhus infections (relative risk, 7.537; 95\% CI, 1.68533.715) $(P=0.002)$, and October was very strongly associated with scrub typhus infections (relative risk, 14.421; 95\% CI, 4.015-51.794) $(P=0.000)$ (Table 4). The phylogenetic analysis results for the $56-\mathrm{kDa}$ TSA gene of $O$. tsutsugamushi are shown in Fig. 2. Two nucleotide sequences were obtained from chiggers in 2017 and deposited to GenBank (L. scutellare: MK451703, L. palpale: MK451704). One sequence (MK451703) was related to the Karp cluster (percent similarity: 89.5-91.7\%) (Fig. 2) (Table 5), and the other sequence (MK451704) was homologous to the Shimokoshi strain (88.7\%) (Fig. 2) (Table 5).
Table 5. The number and percents of chiggers, by month, colleceted from A. agrarius and M. regulus in the Republic of Korea from September 2014 to August 2018 that were positive for Orientia tsutsugamushi

\begin{tabular}{lccccc}
\hline Month & $\begin{array}{r}\text { No. of } \\
\text { tested }\end{array}$ & $\begin{array}{c}\text { No. of } \\
\text { Positive } \\
(\%)\end{array}$ & $\begin{array}{c}P \text {-value } \\
(0.05)\end{array}$ & $\begin{array}{c}\text { Relative } \\
\text { risk }\end{array}$ & $\begin{array}{c}95 \% \\
\text { confidence } \\
\text { interval }\end{array}$ \\
\hline October & 950 & $11(1.16)$ & 0.000 & 14.421 & $\begin{array}{c}4.051- \\
51.794\end{array}$ \\
Other months & 3,696 & $3(0.08)$ & - & 1 & - \\
Total & 4,646 & - & - & - & - \\
\hline
\end{tabular}

*January, February, March, April, May, June, July, August, September, November, December.

\section{DISCUSSION}

O. tsutsugamushi is endemic in Korea throughout the year, and personal protection should be reported to prevent chigger bites, especially a month before the onset of increased numbers of human infections.

Although O. Tsutsugamushi was present in rodents throughout the year, analysis of $O$. tsutsugamushi and the monthly factors in rodents did not demonstrate any association (Table 1). However, this study showed a lower prevalence of infection in rodents than that observed in other studies [15-20]. Because previous studies have only been conducted seasonally (spring, fall), they do not reflect the monthly O. tsutsugamushi antibody prevalence in rodents.

The monthly distribution of chiggers revealed a particularly high prevalence during the fall season (Fig .1), when the incidence of scrub typhus patients was the highest in the population of the country. However, O. tsutsugamushi positive chiggers were unexpectedly found to occur throughout the year, except during summer. Chiggers were first observed in September and a sharp increase in the mean number of chiggers was observed in October, after which the number of chiggers declined to low numbers in December and persisted until July. Therefore, people should be especially aware of the risks associated with outdoor activities in the spring and fall. There have been reports of scrub typhus onset in humans during spring when a large number of chiggers are present; the increased incidence of spring scrub typhus has also raised concerns related to global warming [17].

The prevalence of $O$. tsutsugamushi in chiggers was most commonly associated with $L$. scutellare, and is consistent with L. scutellare as the primary vector of $O$. tsutsugamushi in Korea $[4,5,21]$. Chigger species as a risk factor for rodents was not as- 


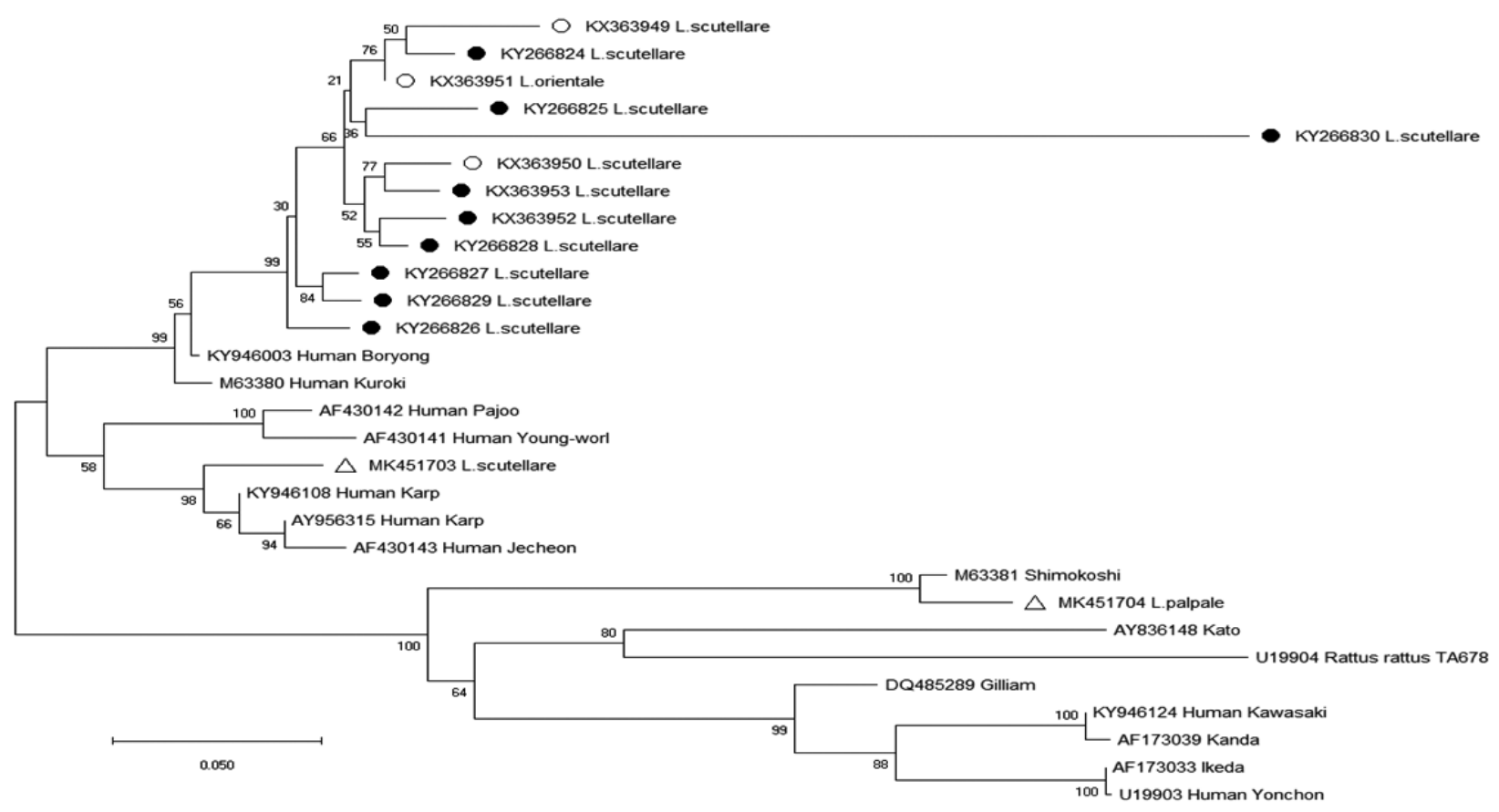

Fig. 2. Phylogenetic tree of Orientia tsutsugamushi based on the 56-kDa gene fragment sequences detected in chiggers in the Republic of Korea from September 2014 to August 2018; open circles, (O) (KX363949-KX363951) [14], and closed circles, (•) (KX363952KX363953, KY266824-KY266830) [13], indicate sequences obtained by Park in previous surveys from 2014-2016 [13,14]; open triangles, $(\triangle)$ (MK451703, MK451704), indicate sequences newly identified in 2017. The phylogenetic tree was constructed via the neighbor-joining method with Kimura's 2-parameter model (1,000 bootstrap replicates) using Mega X. The GenBank accession numbers for O. tsutsugamushi are indicated for each sequence.

sociated with $O$. tsutsugamushi infections, and $O$. tsutsugamushi infections were found to occur in rodent species other than the dominant species collected A. agrarius. Leptotrombidium scutellare showed a strong association with $O$. tsutsugamushi infection (relative risk, $7.537 ; P=0.002)$. Although $L$. scutellare is a known vector of $O$. tsutsugamushi in Korea [6], it showed a stronger association with $O$. tsutsugamushi infection during October (relative risk, 14.421; $P=0.002$ ). According to the KCDC, patient incidence reports were the highest in November [3]. Our results, supported by the incubation period (7-30 days) of scrub typhus in humans [8], which correspond to the time interval from infection to visiting the hospital to receiving treatment after symptom development.

In a study by Park in 2014-2016 [13,14] conducted in the same region, the O. tsutsugamushi genotype for the 56-kDaTSA gene detected in L. scutellare belonged to the Boryong strain, whereas other genotypes (Karp, Shimokoshi) were identified in 2017. Although there wasonly one unique type, the Shimokoshi strain was identified in L. palpale.

In conclusion, these results indicate that people must be aware of the risks of the exposure to chiggers that are vectors of
O. tsutsugamushi, especially during October when transmission is the highest. According to our study, soldiers, farmers, and other people that spend time outdoors belong to high-risk group sand should be careful when performing outdoor activities, especially during October. To establish effective preventive measures for $O$. tsutsugamushi infections, long-term data on meteorological and ecological factors are needed. Studies conducted in additional areas might increase the reliability of disease prediction throughout Korea.

\section{ACKNOWLEDGMENTS}

This study was financially supported by the Health and Environment Research institute (HERI) of Gwang-ju, Korea. This was a joint research project between HERI of Gwang-ju and Chonnam National University.

\section{CONFLICT OF INTEREST}

We have no conflict of interest related to this work. 


\section{REFERENCES}

1. Fedrow AL, Lehman ML, Kelly DJ, Mullins K, Maina AN, Stewart RL, Ge H, Jonh H, Jiang J, Richards AL. A review of scrub typhus (Orientia tsutsugamushi and related organisms): then, now and tomorrow. Trop Med Infect Dis 2018; 3: 8.

2. Xu G, Walker DH, Jupiter D, Melby PC, Arcari CM. A review of the global epidemiology of scrub typhus. Plos One Neg Trop Dis 2017; 11: e0006062.

3. Korea Centers for Disease Control and Prevention. Disease Web Statistics System [Internet]; [cited 2018 January 1]. Available from: http://is.cdc.go.kr/dsta/index.jsp.

4. Song HJ. Environmental survey on the vectors and hosts of tsutsugamushi disease in Jeonnam province, Korea. Korea J Vet Serv 2012; 35: 183-189.

5. Lee IY, Song HJ, Choi YJ, Shin SH, Choi NK, Kwon SH, Shin EH, Pack C, Kim HC, Klein TA, Park KH, Jang WJ. Larval chigger mites collected from small mammals in 3 provinces, Korea. Korean J Parasitol 2014; 52: 225-229.

6. Lee SH, Lee YS, Lee IY, Lim JW, Shin HK, Yu JR, Sim S. Monthly occurrence of vectors and reservoir rodents of scrub typhus in an endemic area of Jeollanamdo, Korea. Korean J Parasitol 2012; 50: 327-331.

7. Takhampunya R, Tippayachai B, Promsathaporn S, Leepitakrat S, Monkanna T, Schuster AL, Melendrez MC, Paris DH, Richards $\mathrm{AL}$, Richardson JH. Characterization based on the 56-Kda typespecific antigen gene of Orientia tsutsugamushi genotypes isolated from Leptotrombidium mites and the rodent host post infection. Am J Trop Med Hyg 2014; 90: 139-146.

8. Kim DM, Kim KY, Man HS, Kweon SS, Park NY, Ryu SY. Risk factors for human infection with Orientia tsutsugamushi: a casecontrol study in Korea. Clin Microbiol Infect 2017; 14: 174-177.

9. Wei Y, Luo L, Jing Q, Le X, Huang U, Xial X, liu L, Wu X, Yang Z. A city park as a potential epidemic site of scrub typhus: a casecontrol study of an outbreak in Guanzhou, China. Parasit Vectors 2014; 7: 513.

10. Wei Y, Huang Y, Li X, Ma Y, Tao X, Wu X, Yang Z. Climate variability, animal reservoir and transmission of scrub typhus in southern China. PLoS Negl Trop Dis 2017; 11: e0005447.

11. Ree HI, Chang WH, Kee SH, Lee IY, Jeon SH. Detection of Orientia tsutsugamushi DNA in individual trombiculids using polymerase chain reaction in Korea. Med Entomol Zool 1997; 48: 197-209.
12. Ree HI. Fauna and key to the chigger mites of Korea (Acarina: Trombiculidae and Leeuwenhoekiidae). Korean J Sys Zool 1990; 6: 57-70.

13. Park JW, Kim SH, Park DW, Jung SH, Park HJ, Seo MH, Song HJ, Lee JY, Kim DM, Kim CM, Gill BC, Jeong HJ, Lee JM, Ha DR, Kim ES, Chung JK. Molecular epidemiology of an Orientia tsutsugamushi gene encoding a 56-kDa type-specific antigen in chiggers, small mammals, patients from the Southwest region of Korea. Am J Trop Med Hyg 2018; 98: 616-624.

14. Park JW, Chung JK, Kim SH, Cho SJ, Ha YD, Jung SH, Park HJ, Song HJ, Lee JY, Kim DM, Pyus Jah, Ha DR, Kim ES, Lee JI. Seroepidemiological survey of zoonotic disease in small mammals with PCR detection of Orientia tsutsugamushi in chiggers, Gwangju, Korea. Korean J Parasitol 2016; 54: 307-313.

15. Kim HC, Lee IY, Chong SU, Richards Al, Gu SH, Song JW, Lee JS, Klein TA. Serosurveillance of scrub typhus in small mammals collected from military training sites near the DMZ, northern Gyeonggi-do, Korea, and analysis of the relative abundance of chiggers from mammals examined. Korean J Parasitol 2010; 48: 237-243.

16. Lin PR, Tsai HP, Weng MH, Lin HC, Chen KC, Kuo MD, Tsui PY, Hung YW, Hsu HL, Liu WT. Field assessment of Orientia tsutsugamushi infection in small mammals and its association with the occurrence of human scrub typhus in Taiwan. Acta Trop 2014; 131: 117-123.

17. Min C, Guo H, Tang T, Wang C, Li X, Pan X, Tang J. Spring scrub typhus, people's Republic of China. Emerg Infect Dis 2006; 12: 1463-1465.

18. Teppawar R, Patii A, Chaudhari S, Shinde S, Kolte S, Bhoyar A, Gilche Vijay. Zoonotic importance of rodents and their vectors in relation to perpetuation of scrub typhus in population. J Entomol Zool 2019; 7: 60-64.

19. Takhampunya R, Korkusol A, Promsathaporn S, Tippayachao B, Leepirakrat S, Richard AL, Davidson SA. Heterogeneity of Orientia tsutsugamushi genotypes in field-collected trombiculid mite from wild-caught small mammals in Thailand. PLoS Negl Trop Dis 2018; 12: $\mathrm{e} 0006632$.

20. Chaisiri K, Cosson JF, Morand S. Infection of rodents by Orientia tsutsugamushi, the agent of scrub typhus, in relation to land use in Thailand. Trop Med Infect Dis 2017; 2: 53.

21. Elliott I, Pearson I, Dajal P, Thomas NV, Roberts T, Newton PN. Scrub typhus ecology: a systematic review of Orientia in vectors and hosts. Parasit Vectors 2019; 12: 513. 